\title{
A Smart Sensor with Hyperspectral/Range Fovea and Panoramic Peripheral View
}

\author{
Tao Wang ${ }^{1,2}$, Zhigang Zhu ${ }^{1,2}$ and Harvey Rhody ${ }^{3}$ \\ ${ }^{1}$ Department of Computer Science, The City College of New York \\ $138^{\text {th }}$ Street and Convent Avenue, New York, NY 10031 \\ ${ }^{2}$ Department of Computer Science, The CUNY Graduate Center \\ 365 Fifth Avenue, New York, NY 10016 \\ Email: \{zhu, twang\}@cs.ccny.cuny.edu \\ ${ }^{3}$ Center for Imaging Science, Rochester Institute of Technology \\ 54 Lomb Memorial Drive, Rochester, NY 14623-5604 \\ Email: rhody@cis.rit.edu
}

\begin{abstract}
We propose an adaptive and effective multimodal peripheral-fovea sensor design for real-time targets tracking. This design is inspired by the biological vision systems for achieving real-time target detection and recognition with a hyperspectral/range fovea and panoramic peripheral view. A realistic scene simulation approach is used to evaluate our sensor design and the related data exploitation algorithms before a real sensor is made. The goal is to reduce development time and system cost while achieving optimal results through an iterative process that incorporates simulation, sensing, processing and evaluation. Important issues such as multimodal sensory component integration, region of interest extraction, target tracking, hyperspectral image analysis and target signature identification are discussed.
\end{abstract}

Keywords - Hyperspectral imaging, panoramic vision bio-inspired sensing, target tracking.

\section{Introduction}

Recently, a great deal of effort has been put into adaptive and tunable multi-spectral or hyper-spectral sensor designs with goals to address the challenging problems of detecting, tracking and identifying targets in highly cluttered, dynamic scenes. Representative large programs include: the DARPA's Adaptive Focal Plane Array (AFPA) Program [1], ARL's Advanced Sensor CTA [2], and NSF's Center for Mid-Infrared Technologies for Health and the Environment [3]. Even commercial real-time, spectral tunable, polaralization-enabled, hyperspectral imaging (HSI) systems are available [4]. However, today's HSI system are limited to scanning mode detection, and are usually large, complex, power hungry and slow. The ability to perform HSI in a staring mode is critical to real-time targeting mission. This brings up conflicting requirements for real-time search with the ability to detect and identify difficult and hidden targets using hyperspectral information, while staying within the processing time and size available suited to small platforms. In addition, the limited field of view (FOV) of conventional sensor design does not satisfy the requirements of large area search. We propose a novel single sensor design that has both a panoramic view and a hyperspectral/range fovea, and that integrates sensing and processing for real-time target detection. It is an extension to the conventional foveated-peripheral system: 1) It has a panoramic view instead of just a wide-angle view. 2) It is capable of 3D and hyperspectral data acquisition instead of just high-resolution color vision in the foveated region. Therefore, this sensor design is inspired by the biological vision systems of human eyes, but it does not merely mimic human vision. It uses dual-panoramic scanners to detect moving targets and to aid active control of the hyperspectral/range fovea to acquire needed signatures of targets at various locations for achieving real-time hyperspectral and range imaging.

Another important feature of the work is to use a realistic hyperspectral simulation tool to test our sensor designs in various scenarios, which allows us to more effectively refine our sensor parameters. In addition, it can further help us to reduce the cost of fabrication of a sensor design that might not yet meet the requirements of real-world applications before the images "captured" by the sensor are processed for the applications. Using realistic scene simulation and image generation using the designed sensor parameters, various algorithms can be applied to the generated images and then the sensor design can be evaluated and further refined. In this paper, issues such as sensor designs, peripheral background modeling, and target signature acquisition will be addressed in detail. This design and the related data exploitation algorithms 


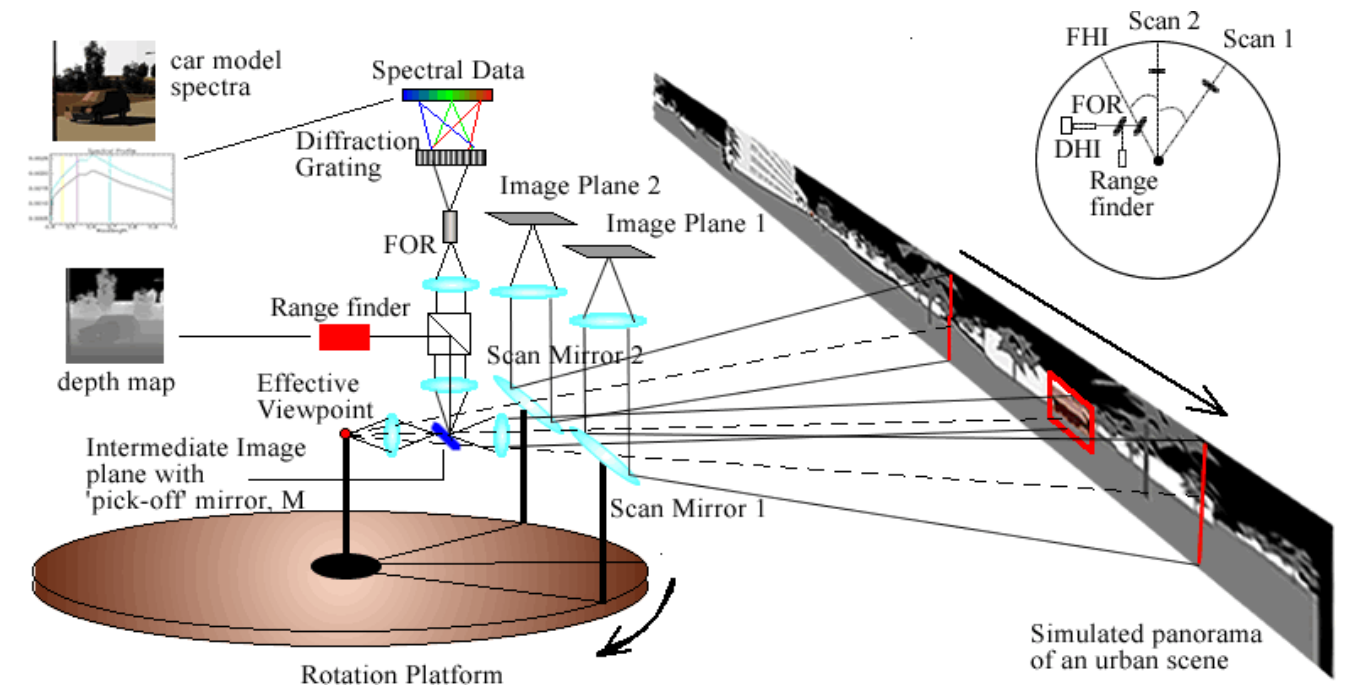

Fig. 1. The design concept of the DPSHRF. The dash lines indicate the single viewpoint of both the foveal hyperspectral imager and the two line scanners.

will be simulated and evaluated in our general data simulation framework.

The paper is organized as the following. Section 2 shows the design of the bio-inspired adaptive multimodal sensor platform - the dual panoramic scanners with hyperspectral/range fovea (DPSHRF) for the task of tracking moving targets in real time. Section 3 describes the simulation environment for implementing our simulation approach, and the parameter configuration of the sensor platform. Section 4 presents the image exploitation algorithms for detecting and tracking moving targets, and spectral classification in recognizing the moving objects. Conclusions and discussions will be provided in Section 5.

\section{A Bio-Inspired Sensor Design}

The data volumes in consideration have two spatial dimensions ( $\mathrm{X}$ and $\mathrm{Y}$ ), a spectral dimension ( $\mathrm{S}$, from a few to several hundred), and a time dimension (T). This four dimensional (4D) image in X-Y-S-T may be augmented by a 2D range image (in the $X Y$ space). Ideally, a sensor should have 360-degree full spherical coverage, with high spatial and temporal resolution, and at each pixel have full range of spectral and range information. However, this type of sensor is difficult to implement because of the enormous amount of data that must be captured and transmitted, most of which will eventually be discarded. Therefore, particularly for real-time applications, every collection must face fundamental trade-offs such as spatial resolution vs spectral resolution, collection rate vs SNR, field-of-view vs coverage, to name a few examples.

Understanding the trade-offs and using algorithms that can be adapted to changing requirements can improve performance by enabling the collection to be done with maximum effectiveness for the current task. To break the dilemma between FOV and spatial/spectral resolution, we investigate a bio-inspired data collection strategy, which can achieve real-time imaging with a hyperspectral/range fovea and panoramic peripheral view. This is an extension of the functions of human eyes that have high-resolution color vision in the fovea and black-white, low-resolution target detection in the wide field-of-view peripheral vision. In our design, the fovea is enhanced by HSI and range information, and the peripheral vision is extended to panoramic FOV and has adaptive spectral response rather than just black-white.

Our proposed sensor platform, the dual-panoramic scanners with a hyperspectral/range fovea (DPSHRF) (Fig. 1), consists of a dual-panoramic (omnidirectional) peripheral vision and a narrow FOV hyperspectral fovea with a range finder. This intelligent sensor works as the follows. In the first step, two panchromatic images with 360-degree FOV are generated by rotating two line scanners around a common rotating axis, pointing apart to two slightly different directions. The angle difference between the two scanners can be adjusted for detecting and tracking moving targets with different velocities and distances. An initial angle is used at the beginning. Then the detecting results from the two scans can determine what the new angle difference should be - either decreased if a target is moving too fast, or increased if the target is moving too slow. There are two advantages of using line scanners that will be further amplified. First, a line scanner can have a full 360-degree horizontal FOV. Second, resulted images are inherently registered.

Moving targets can then be easily and quickly determined by the differences of the two panoramic 
images generated from two scanners. The next position and the time of a moving target can be estimated from the difference of two regions of interest (ROIs) that include the target. In real-time processing, the comparison is started whenever the second scan reaches the position of the first scan, therefore, only a small portion of panoramic images is used before full-view panoramas are generated. The detail of the target detection processing algorithm will be discussed in Section 4.

Then, we can turn the hyperspectral/range fovea with a specific focal length calculated based on the size of the object, and to the predicted region that includes the moving target. Thus, hyperspectral/range data is recorded more efficiently for only the ROIs that include possible moving targets. The two line scanners and the hyperspectral/range imager are aligned so that they all share a single effective viewpoint. The spectral data can be efficiently recorded with a foveal hyperspectral imager (FHI) [6] which maps a 2D spatial image into a spatial 1D image. This is implemented by using a micro mirror as a fovea that intercepts the light onto a beam splitter for generating co-registered range-hyperspectral images using a ranger finder and the FHI. The FHI consists of a fiber optical reformatter (FOR) [7] forms a 1D array onto a dispersive hyperspectral imager (DHI) [8] which produces a 2D hyperspectral data array with one dimension as spatial and the other as spectral. The spatial resolution of the FOR is determined by the diameters of optical fibers which are controlled during the optical design process. The blurring effect from cross-coupling of optical fibers is not significant magnitude as shown in [9]. Finally, a co-registered spatial-spectral/range image is produced by combining with the panchromatic images which are generated by the dual-panoramic scanners.

In summary, this sensor platform improves or differs from previous designs $[2,6,9]$ in literature in four aspects: (1) A dual scanning system is designed to obtain moving targets in a very effective and efficient manner. A panoramic view is provided instead of a normal wide-angle view.

(2) An integration of range and hyperspectral fovea component is used for target identification.

(3) The dual-panoramic scanners and the hyperspectral/range fovea are co-registered.

(4) Active control of the hyperspectral sensor is added to facilitate signature acquisition of targets of various locations that can only be determined in real-time.

\section{Scene Simulation and Sensor Modeling}

The sensor design concept is tested though the simulation tool DIRSIG. Various broad-band, multi-spectral and hyperspectral imagery are generated through the integration of a suite of first principles based radiation propagation sub-models [10]. Before performing scene simulation and sensor modeling, we need to set up different scenarios and configure the sensor parameters. One of the complex scenarios we constructed including 4 cars having exactly same shapes and 3 different paints moving to different directions with various speeds (Fig. 2). All four cars will pass through the cross section at the bottom corner of the main building in the scene at a certain time. Various behaviors of the moving vehicles such as simple moving, overtaking, passing through, and etc., are monitored by our sensor platform which is placed in front of the main building. The scan speed of each line scanner can be set from $60 \mathrm{~Hz}$ to $100 \mathrm{~Hz}$ selectable, thus one entire $360^{\circ}$ scan take from 6.0 seconds down to 3.6 seconds. This time constraint is not a problem for real-time target detection since detection and scanning are continuous and simultaneous. The number of pixels per line in the vertical direction is set to 512 to match the horizontal scanning resolution. Few selected spectral bands are captured by dual line scanning. The focal length is fixed at $35 \mathrm{~mm}$ for both line scanners, and the angle between the pointing directions of the two scanners is $10^{\circ}$ so that the time the second scan reaches the position of the first scan is only about $0.1 \mathrm{~s}$. In theory, the time difference between two scans should be much less than one second to avoid a lot of uncertainty of action changes in moving vehicles. Two scanners are used so that (1) the more accurate direction and the focal length of the hyperspectral fovea can be estimated; and (2) moving target detection can still be performed when background subtraction using a single scanner fails due to cluttered background, multiple moving targets, and the ego-motion of the sensor platform. The focal length of the hyperspectral imager is automatically adjusted according to the target detection results generated from the two line scanners. To simulate the hyperspectral imager, we use a frame array sensor with small spatial resolution at $70 \times 70$ for the hyperspectral data, and the ground truth range data provided by DIRSIG are transformed into range images. The spectral resolution is $0.01 \mu \mathrm{m}$ ranged from $0.4 \mu \mathrm{m}$ to $1.0 \mu \mathrm{m}$. Different portion of bandwidth can be selected and determined by analyzing the model spectral profile.

The simulation will enable a close investigation of intelligent sensor designs and hyperspectral data selection and exploitation for user designated targets. The DIRSIG simulation environment allows us to use an iterative approach to multimodal sensor designs. Starting with user and application requirements, various targets of interest in different, cluttered background can be simulated using the scene-target simulation tools in the DIRSIG. Then the adaptive multimodal sensor that has been designed can be modeled using the sensor modeling tools within the DIRSIG, and multimodal sensing data (images) can be generated. Target detection/identification, background modeling and multimodal fusion algorithms will be run on these simulated images to evaluate the overall 


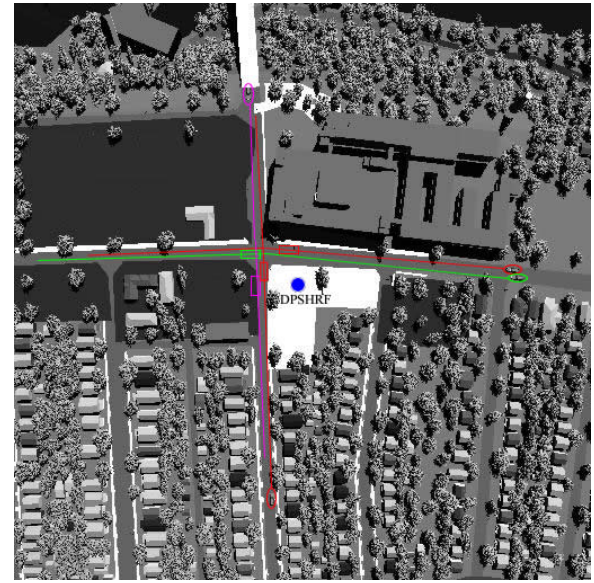

Fig. 2. A simulated urban scene image captured at latitude $=43.0^{\circ}$ and longitude $=77.0^{\circ}, 1000$ meters above. The ellipses show the initial state of the four cars. The rectangles show the state where those cars move after 12s. The "DPSHRF" sensor (in blue dot) is placed in front of the main large building. The simulated scene is captured at $8 \mathrm{am}$ in a typical summer day.

performance of the automated target recognition, and to investigate the effectiveness of the initial multimodal sensor design. The evaluations of the recognition results against the given "ground-truth" data (by simulation) can provide further indicators for improving the initial sensor design, for example, spatial resolution, temporal sampling rates, spectral band selection, the role of range information and polarization, etc.. Finally, a refined sensor design can again be modeled within the DIRSIG to start another iteration of sensor and system evaluation.

\section{Data Exploitation and Adaptive Sensing}

The basic procedure for active target detection and tracking is as follows. A few selected spectral bands are used to initialize the detection of targets either based on motion detection or scene/target properties in prior scenarios. Then, for the potential interesting targets, the fovea turns to each of them to get a high-resolution, hyperspectral image with range information. This can be done in real-time so that tracking of one target and switching between multiple candidates is made possible. Finally, the signatures of the targets can be obtained by automatically analyzing the hyperspectral data in the fovea and by selecting the most relevant bands for such targets. This kind of function needs the active control of the sensor to fuse the peripheral and fovea vision in an efficient manner. In the following, we elaborate the principle by using some commonly used algorithms in target detection, tracking and identification, using our bio-inspired multimodal sensor.

\subsection{Detection and tracking in peripheral views}

The first step is to find ROIs that possibly contain moving targets (Fig 3). Simple background subtraction between a scanned image and a background image is not sufficient because the panoramic background (with trees, building, etc.) may change due to illumination changes over a large span of time. The advantage of using the two consecutive scanners is the ability to quickly detect a moving target in real time using "frame difference" without producing too much noise from the background. Further, a morphological noise removal technique [11] is applied to remove small sparse noises with the opening operation and fill small holes with the closing operation. However, the results from "frame difference" cannot provide accurate location and size information of the moving targets. Therefore, bounding boxes are defined from the "frame difference" results to mask off those background regions for background subtraction, which can provide more accurate location and size information of the moving targets. Fig. $3 \mathrm{c}$ shows some bounding boxes that can be used as masks for performing the background subtraction of each individual panoramic scan. The threshold is set very low since we are interested in any changes in motion comparing to the relative static background. Of course, false alarms can also be generated by events such as the change of a large shadow, but this can be verified once we captured the hyperspectral image. The background image is updated for only those pixels belonging to the background after each 360-degree rotation, thus moving object extraction is maintained over time. At every rotation, each of the two line scanners will generate a sequence of 1D image lines that are combined to generate the panorama. Thus, registration problems can be avoided with the stabilized line scanners. Real-time target detection can be achieved since the scanning and detection are performed simultaneously and continuously.

The next step is to estimate the region of the next position that may contain a target once the two ROIs of the same target are found at two different times resulting from two different scans (Fig. 3d). The location and size differences of the two regions can determine the relative bearing angle of the hyperspectral/range fovea imager to zoom on the moving target. The position of extracted region from the dual-scans indicates which direction the target is moving to. Also, the size of two regions can indicate whether the target is moving closer to the sensor or farther. Therefore, we can calculate the next position where the target will be. Then, the ratio of the previous two regions can be used to estimate the new focal length of the hyperspectral imager.

The angle difference of two scans for two ROIs at different times $t_{i}$ and $t_{i+1}$, can be used to predict the position of the next ROI having the moving target at the time, $t_{i+2}$, when the hyperspectral/range imager can be in 

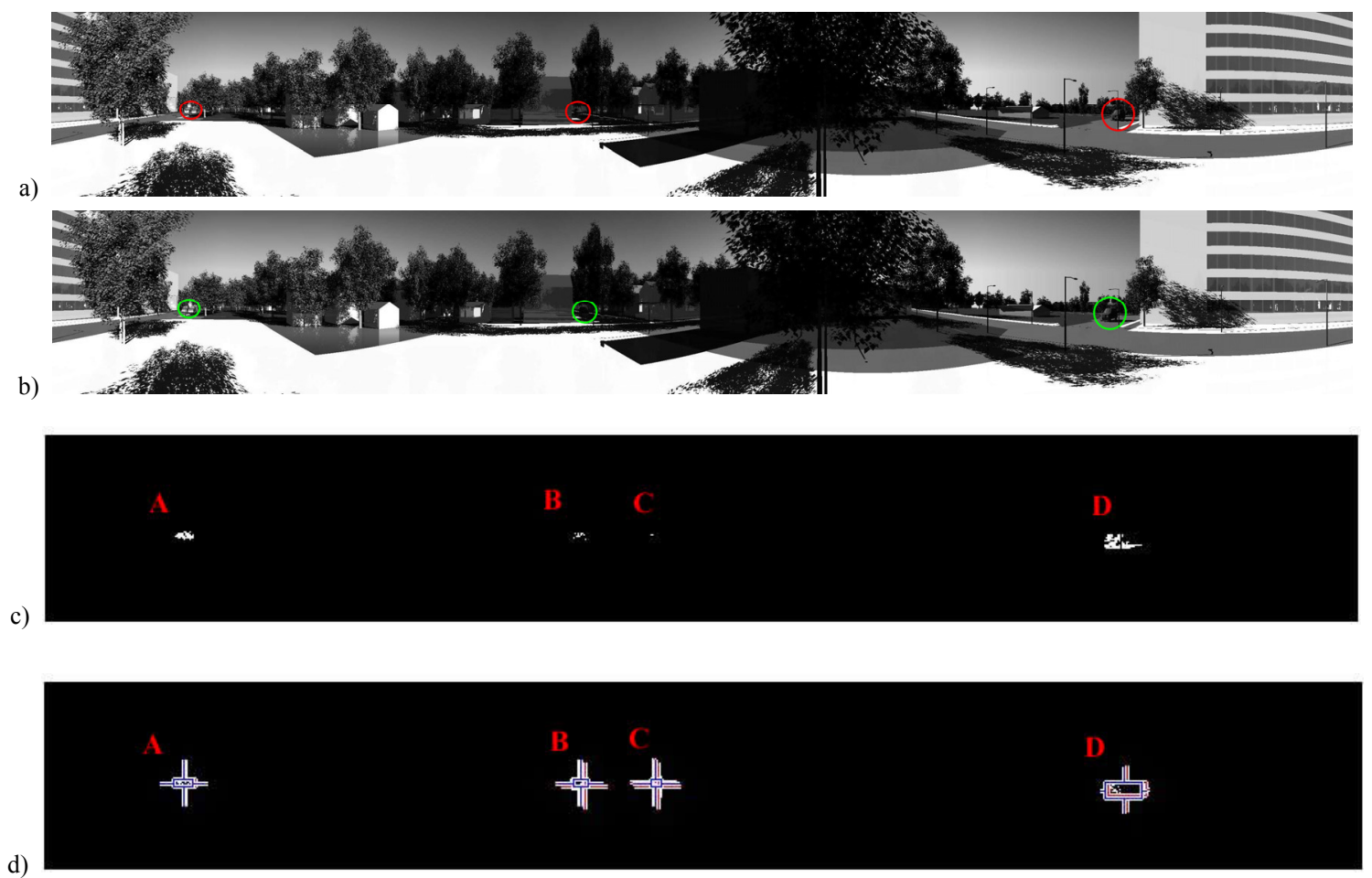

Fig. 3. All $360^{\circ}$ panoramic images $(512 \times 3600)$ shown here are integrated from vertical scan lines captured by the dual-panoramic scanners. a). Panoramic image from the first scanner, with the moving targets indicated inside red circles. b). Panoramic image from the second scanner, again the same moving targets indicated inside green circles. c). frame difference between a and b, group of ROIs are labeled. d). background subtraction from two scans inside boundaries defined by c. Red rectangles showed ROIs from first scan, blue rectangles showed ROIs from second scan. (Close-up view of each labeled region can be seen clearly in Fig. 4).

place. Therefore, given the time $t_{i+2}$, we can estimate the panning and tile angles of the hyperspectral/range imager. Note that only the angles relative to the center of a region are needed. The turning angles (i.e., panning and tilting) of the hyperspectral/range imager should be:

$$
\theta_{t_{i+2}}^{(x, y)}=\theta_{t_{i+1}}^{(x, y)}+\frac{t_{i+2}^{(x, y)}-t_{i+1}^{(x, y)}}{t_{i+1}^{(x, y)}-t_{i}^{(x, y)}}\left(\theta_{t_{i+1}}^{(x, y)}-\theta_{t_{i}}^{(x, y)}\right)
$$

where the superscript $\mathrm{x}$ and $\mathrm{y}$ correspond to the panning angle (in the $\mathrm{x}$-direction) and the tilting angle (in the $\mathrm{y}$-direction), respectively. The angle $\theta_{t_{i}}$ corresponds to the angle position of a ROI at a time $t_{i}$ as shown in Fig. 3 . The focal length of the hyperspectral/range fovea is inversely proportional to the desired FOV of the hyperspectral/range imager, $\alpha$, in order to have the target in the full view of the FOV. The FOV angle can be estimated as

$$
\alpha=\frac{R_{t_{i+2}}}{P^{l}}
$$

where $R_{t_{i+2}}$ is the predicted size of the target region at $t_{i+2}$, and $P^{l}$ is the number of scanning lines per radius. The relationship between $R_{t_{i+2}}$ and the previous two regions of the same target at different times can be expressed as

$$
\frac{R_{t_{i+1}}}{R_{t_{i}}}\left(t_{i+1}-t_{i}\right)=\frac{R_{t_{i+2}}}{R_{t_{i+1}}}\left(t_{i+2}-t_{i+1}\right)
$$

Then a hyperspectral foveal shot of a ROI from the calculation can be taken. Thus, hyperspectral/range data is recorded in a more efficient way, only for ROIs. It is possible for some regions to be identified that do not have true moving targets inside. Then the hyperspectral classification in next step can verify this situation.

\subsection{Target classification using $3 D$ and HSI fovea}

Targets can be classified based on hyperspectral measurements, shape information, and the integration of both. There has been a lot of work in recognizing objects using 3D shape information [e.g., 12, 13]. Here we will only describe how to use a target's depth information and 
the information of its background to perform better hyperspectral classification.

Recognizing a target needs to compare the target's spectrum associated with each pixel to its training spectrum. In our experiments, a spectral library was pre-built with some existing models. Various vehicles with different colors and shapes can be imported and tested in the simulation scene. In the particular scenario in Fig. 2, four cars having the same shape but different paints are modeled. Two are red, one is brown and one is black. Initial spectral signatures of the four cars were captured from different angles in the same background. The capturing angles and surroundings are important and need to be considered carefully because those factors can significantly affect the effective radiance reaching the sensor, $L(l, \theta, \phi, \lambda)$, where $l$ is the slant range from sensor to target, $\theta, \phi$ and $\lambda$ are the zenith angle, the azimuth angle and the wavelength, respectively. The general expression for $L$ is more complex and fully described in [14]. However, we can simplify $L$ if we are only interested in the reflective (visible) bands, the general equation can be further expressed as:

$$
L(l, \theta, \phi, \lambda)=f\left(L_{s}(l, \sigma, \lambda), L_{d s}(\theta, \phi), L_{b s}(\theta, \phi, \lambda), L_{u s}(l, \theta, \lambda)\right)(4)
$$

where $\sigma$ is the angle from the normal to the target to the sun, $L_{s}$ is the solar radiance, $L_{d s}$ is the downwelled radiance from the sky due to the atmospheric scattering, $L_{b s}$ is the spectral radiance due to the reflection from background objects, and $L_{u s}$ is the scattered atmospheric path radiance along the target-sensor line of site.

In the training stage, the background is known and fixed, thus $L_{b s}$ can be cancelled out. The angles of the sun to the target and the of target to the sensor are known, thus we can keep this information and estimate a new spectral profile of the model target once we need to monitor a new target at a different time. $L_{d s}$ and $L_{u s}$ can also affect the initial spectral profile if the weather condition changes significantly. In the current experiments, we only use one atmospheric dataset which can also be replaced and changed in the simulation in the future. After handling all reflective variants, various endmembers that represent the spectral extremes that best characterize a material type of a target were selected, and their spectral curves were stored in the spectral library database. We used the sequential maximum angle convex cone (SMACC) [15] to extract spectral endmembers and their abundance for every model target. In comparison to the conventional pixel purity index (PPI) [16] and N-FINDER [17], SMACC is a much faster and more automated method for finding spectral endmembers. Simply speaking, SMACC first finds extreme points or vectors that cannot be represented by a positive linear combination of other vectors in the data as a convex cone, and then a constrained oblique projection is applied to the existing cone to derive the next endmembers. The process is repeated until a tolerance value is reached, for example, max number of endmembers. Each endmember spectrum, defined as $H$, can be presented mathematically as a combination of the product of a convex 2D matrix contains endmember spectra as columns and a positive coefficient matrix:

$$
H(c, i)=\sum_{k}^{N} R(c, k) A(k, j)
$$

where $i$ is the pixel index, $j$ and $k$ are the endmember indices, and $c$ is the spectra channel index. Some endmembers might have less spectra differences in term of redundancy. Those can be coalesced based on a threshold so that the most extreme spectra are identified and used to represent the entire coalesced group of endmembers.

In the testing stage, the same target spectra may be varied in different conditions such as various surface orientations and surroundings. However, the significant spectral signature of a target can be estimated and maybe further corrected with the help of range information produced from a ranger finder. Knowing the angles of the sun and the sensor, the depth map (i.e. range data) can indicate whether the information of a background object close to the target should be counted when processing the target spectra. The result spectra will have similar shape but the magnitudes will be still different due to the variations of illumination intensities and directions. A spectral angle mapper (SAM) [18] algorithm is used to match the target spectra to reference spectra. The SAM is insensitive to illumination and albedo effects. The algorithm determines the spectral similarity between two spectra by calculating the angle between the spectra and treating them as vectors in a space with dimensionality equal to the number of bands [18]. Smaller angles represent closer match. The depth information and the relative location of the sun and the sensor can determine whether a target spectra should be adjusted by the surrounding spectra when performing classification. As a result, each pixel is classified either to a known object if the target spectrum is matched with the library spectrum of that object, or to an unknown object, for instance, the background. To distinct multiple objects from database, the results from different group of endmembers of different targets are compared.

\subsection{Experimental Results}

Fig. 4 shows the processed results for the following four cases: A) Multiple targets with different spectral signature. B) A target is under a shadow cast by trees. C) There is no moving target (thus a false alarm). D) Only one side of the target spectral signature can be acquired and the other side can not be determined due to the insufficient reflectance of the sun light and the surroundings. Various solutions can be possible, for example: 1) to place the sensor platform at 


\begin{tabular}{|c|c|c|c|c|c|c|c|c|c|}
\hline Index & ROIs & $\begin{array}{c}\text { Fovea } \\
\text { parameters }\end{array}$ & $\begin{array}{c}\text { Fovea Shot } \\
\text { (in RGB) }\end{array}$ & $\begin{array}{c}\text { Sample } \\
\text { Spectral Profile }\end{array}$ & $\begin{array}{c}\text { Spectral Curves } \\
\text { Annotations }\end{array}$ & Depth Map & $\begin{array}{c}\text { SAM } \\
\text { without depth }\end{array}$ & $\begin{array}{l}\text { SAM with } \\
\text { depth }\end{array}$ & Results \\
\hline A & 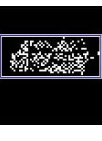 & $\begin{array}{c}\text { Zenith: } 89.0 \\
\text { Azimuth: } 80.0 \\
\text { Focal Length: } \\
245 \mathrm{~mm}\end{array}$ & & 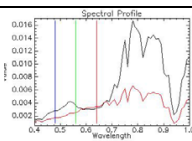 & $\begin{array}{c}\text { Top Curve: } \\
\text { Car on Right } \\
\text { Bottom Curve: } \\
\text { Car on Left }\end{array}$ & & & & $\begin{array}{c}\text { Red Car } \\
\text { Brown Car }\end{array}$ \\
\hline B & 89 & $\begin{array}{c}\text { Zenith: } 88.5 \\
\text { Azimuth } 191.0 \\
\text { Focal Length: } \\
205 \mathrm{~mm}\end{array}$ & & 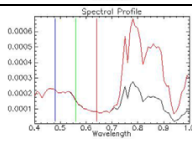 & $\begin{array}{c}\text { Top Curve: } \\
\text { Car not in Shadow } \\
\text { Bottom Curve: } \\
\text { Car in Shadow }\end{array}$ & & $\rightarrow$ & 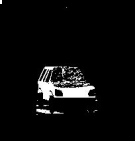 & Red Car \\
\hline $\mathrm{C}$ & 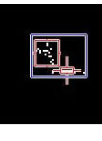 & $\begin{array}{l}\text { Zenith: } 88.5 \\
\text { Azimuth } 220.0 \\
\text { Focal Length }= \\
225 \mathrm{~mm}\end{array}$ & & | & $\begin{array}{c}\frac{\text { Top Curve: }}{\text { Material } 1} \\
\text { Bottom Curve: } \\
\text { Material } 2\end{array}$ & & & & False Target \\
\hline $\mathrm{D}$ & 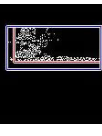 & $\begin{array}{c}\text { Zenith: } 88.0 \\
\text { Azimuth } 330.0 \\
\text { Focal Length: } \\
125 \mathrm{~mm}\end{array}$ & 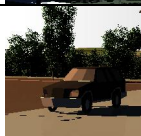 & 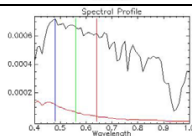 & $\begin{array}{c}\frac{\text { Top Curve: }}{\text { Front body }} \\
\text { Bottom Curve: } \\
\text { Side body }\end{array}$ & & & & Black Car \\
\hline
\end{tabular}

Fig. 4. Processing Result of the simulated urban scene. Each index corresponds to each labeled region in Fig 2d. The column ROIs shows close-up view of result indicated in Fig 2d. Hyperspectral fovea shots demonstrated here with only 3 RGB bands (which are also marked as vertical lines in the sample spectral profile column, in blue, green and red, respectively. Only the significant spectral signatures of targets are shown here. Final mapping results are shown in binary only to indicate the targets and the background. The classification is based on the match result with each model target spectral profile in database.

another position; 2) to reconfigure sensor parameters such as adjust the height and the pointing direction; and 3) to implement a better classification algorithm. Therefore the experimental results can quickly drive feedback to adjust and improve the sensor design and the algorithm implementations. Various scenarios and cases can be constructed and tested in the simulation framework before a real sensor is even made.

One of the useful advantages of the co-registered hyperspectral and range imaging is to use the range information to improve the effectiveness of the hyperspectral measurements. For example, in Fig. 4B, the shadowing of the vehicle (the red car) under the trees can be analyzed by the relation among the location of the sun, the locations of the trees from the panoramic background, and the surface orientations of the vehicle. Considering the depth information, the SAM can be obtained for surfaces of the vehicle under the influence of the tree shadows (therefore looks greenish). In Fig. 4D, the relations between the surfaces orientations of the vehicle (the black car) and the location of the sun can also tell which surfaces are illuminated. Therefore the well-illuminated surfaces (i.e. the top of the car body) can be selected based on the structural information obtained from the range data. The analysis so far is very preliminary but is very promising for future research.

\section{Conclusions and Discussion}

We have described our bio-inspired multimodal sensor design that enables efficient hyperspectral data collection for tracking moving targets in real-time. The real-time hyperspectral/range fovea imaging system further extends the capability of human fovea vision. We have also demonstrated a simulation procedure to evaluate the new senor design, to test algorithms and to actively control the sensor for data selection. Constructing a sophisticated sensor like the one described in the paper is time-consuming and costly, and might not even meet the application requirements after being fabricated. Therefore, we believe the simulation procedure used in the paper is one solution to thoroughly evaluate a new sensor design in that realistic images can be generated in order to test various algorithms before the sensor has been made. By simulation, various components can be reconfigured or replaced for specific situations or tasks. The image processing algorithms described in the paper are designed only to demonstrate the basic idea of effectively capturing hyperspectral data in ROIs based on data exploitation. Needless to say, more sophisticated algorithms need to be developed for more challenging tasks. In addition, we only described one spectral classification method for recognizing the object. More precise and efficient hyperspectral classification routines may be applied.

In the future, we will study two aspects of data processing: range-spectral integration and intelligent spectral band selection. Both issues will be greatly facilitated by our proposed approach with advanced scene and sensor simulation.

Range-spectral integration. There are many factors that need to be considered in correcting the acquired hyperspectral data to reveal the true material reflectance, including source illumination, scene geometry, atmospheric and sensor effects, spectral and space 
resolution, and etc. In the low-altitude airborne or ground imaging cases, the scene geometry is probably the most important factor. Therefore, the design of co-registered hyperspectral and range fovea will provide both spectral and geometry measurements of the $3 \mathrm{D}$ scene in a high resolution, so that a range-aided spectral correction can be performed. Using the DIRSIG tools, we have simulated both hyperspectral images and ranges images for several selected targets with known 3D models and spectral properties, and the next step to derive algorithms to perform spectral correction by the more effective $3 \mathrm{D}$ structure information of the targets given by the range images and the background information given by the panoramic scanners.

Optimal band selection. After the analysis of the hyperspectral data, the most useful wavelengths that can capture the target's signatures can be selected via tunable filtering; and the task of tracking and target recognition will only need to use the few selected bands or a few key features rather than all of the bands. This study will be carried out in several scenarios involving different targets in a challenging background or different backgrounds. We will compare the hyperspectral profiles (i.e. 3D images with two spatial dimensions and a spectral dimension) of various targets against different background materials, and then derive the optimal spectral signatures to distinguish a target from its background. We will also investigate how the range information can be used in improving the effectiveness of signature extraction and target recognition. The DIRSIG target and scene simulation tools could provide sufficient samples as training examples for us to optimal hyperspectral band selection.

\section{Acknowledgements}

This work is supported by AFOSR under the Discovery Challenge Trusts (DCTs) Program, Award \#FA9550-08-1-0199, and in part by AFRL/SN under Award No. FA8650-05-1-1853 and by NSF under Grant No. CNS-0551598.

\section{References}

[1] Stu Horn, DARPA's Adaptive Focal Plane Array (AFPA) Program, Available: http://www.arpa.mil/mto/programs/afpa/index.html

[2] A.C. Goldberg, B. Stann, N. Gupta, 2003, "Multispectral, hyperspectral, and three-dimensional imaging research at the U.S. Army research laboratory", Proceedings of the Sixth International Conference of Information Fusion, 2003. Vol. 1, 2003, pp: 499-506
[3] K. Kincade, "MIRTHE center aims to take mid-IR sensors to new heights. (Optical Sensing)", Laser Focus World, July, 2006.

[4] POC, POC's Real-Time Hyperspectral Imaging, Available: http://www.poc.com/, last visited July, 2008

[5] DIRSIG, http://dirsig.cis.rit.edu/, last visited October, 2008

[6] Fletcher-Holmes, D. W. and A. R. Harvey, 2005. "Real-time imaging with a hyperspectral fovea," J. Opt. A: Pure Appl. Opt. 7, 2005, pp. S298-S302

[7] Fibreoptic Systems Inc. 60 Moreland Rd, Unit A, Simi Valley, CA 93065, USA (http:/www.fibopsys.com/)

[8] Headwall Photonics, Inc., 601 River Street, Fitchburg, MA 01420, USA.(http://www.headwallphotonics.com/)

[9] A. R. Harvey, D W Fletcher-Holmes, Imaging aparatus, GB Patent application 0215248.6, 2nd July 2002

[10] J. R. Schott, S. D. Brown, R. V. Raqueño, H. N. Gross, and G. Robinson, "An advanced synthetic image generation model and its application to multi/hyperspectral algorithm development", Canadian Journal of Remote Sensing, Vol. 25, No. 2, June 1999

[11] P. Soille, "Morphological image analysis: principle and applications", Springer Press, 1999

[12] A. Diplaros, T. Geves, I. Patras, "Combining color and shape information for illumination-viewpoint invariant object recognition". Image Process, Vol. 1, Issue 1, pp.1-11, Jan. 2006

[13] T. M. Start and M. A. Fischler, "Context-based vision: recognizing objects using information from both $2 \mathrm{D}$ and $3 \mathrm{D}$ imagery", Pattern Analysis and Machine Intelligence, IEEE Trans. Vol. 13, Issue 10, Oct 1991

[14] J. R. Schott, "Remote Sensing: the image chain approach, 2nd ed". Oxford, NY: Oxford University Press, 2007.

[15] J. Gruninger, A. J. Ratkowski and M. L. Hoke. "The sequential maximum angle convex cone (SMACC) endmember model". Proceedings SPIE, Algorithms for Multispectral and Hyper-spectral and Ultraspectral Imagery, Vol. 5425-1, Orlando FL, April, 2004.

[16] J. W. Boardman, F.A. Druse, and R.O. Green, "Mapping target signatures via partial unmixing of AVIRIS data." Fifth Annual JPL Airborne Earth Science Workshop, Vol. 1, AVIRIS Workshop, pp. 23-26, 1995

[17] M. F. Winter, "N-FINDR: an algorithm for fast autonomous spectral end-member determination in hyperspectral data." Proceedings SPIE, Vol. 3753, pp. 266-275

[18] F. A. Kruse, et al. 1993, "The spectral image processing system (SIPS) - Interactive visualization and analysis of imaging spectrometer data". Remote Sensing of the Environment, v. 44, p. 145 - 163. 